\title{
On Numerical Contour Integration Round a Closed Contour*
}

\author{
By J. N. Lyness $\dagger$ and L. M. Delves $\ddagger$
}

1. Introduction. In a companion paper [2] referred to here as Paper A, we give an algorithm for locating all the roots of an analytic function $f(z)$ within a region $R$ in the complex plane. This algorithm involves the numerical integration of certain functions round a simple closed contour $C$ which may be a circle or a square. In some forms of the algorithm, it is also necessary to interpolate along the contour. In this paper we discuss the accuracy of the integration and interpolation procedures used, and obtain bounds on the truncation error in the various cases that arise. The theory given here is quite general, and the presentation is independent of Paper A. However, particular attention is given to the special cases needed there.

In a previous paper (Lyness and Moler [3]) the numerical calculation of derivatives of an analytic function was investigated. Briefly, if $f(z)$ is an analytic function whose power series

$$
f(z)=\sum_{j=0}^{\infty} a_{j} z^{j}
$$

has radius of convergence $\bar{R}$ greater than $R$, the Taylor coefficient $a_{j}$ is given by

$$
a_{j}=\frac{1}{2 \pi i} \int_{C} \frac{f(z)}{z^{j+1}} d z ;
$$

$C$ being a simple closed contour lying wholly within the region $|z|=R$ and containing the origin. If we denote by $C_{r}$ the contour $|z|=r$, the approximation to $a_{j}$ based on using an $N$-point trapezoidal rule for the contour integral is $\tilde{a}_{j}^{(N)}$ given by

$$
\begin{aligned}
r^{j} \tilde{a}_{j}^{(N)} & =\frac{1}{N} \sum_{g=1}^{N} \exp (-2 \pi i g j / N) f(r \exp (2 \pi i g / N)), & & j=0,1, \cdots, N-1, \\
\tilde{a}_{j}^{(N)} & =0, & & j=N, N+1, \cdots .
\end{aligned}
$$

A bound on the discretization error in terms of the lower bound $R$ to $\bar{R}$ has been obtained previously (Lyness [4]). There the discretization error

$$
\epsilon_{j, 1}^{(N)}(r)=r^{j} a_{j}-r^{j} \tilde{a}_{j}^{(N)}
$$

was bounded using Schwarz's inequality.

In terms of $(J(R))^{2} \equiv I(R)$;

Received November 9, 1966. Revised February 20, 1967.

* Research sponsored by the U.S. Atomic Energy Commission under contract with the Union Carbide Corporation.

† On leave of absence from the University of New South Wales, Australia. Present address: Argonne National Laboratory, Argonne, Illinois.

$\ddagger$ On leave of absence from the University of Sussex, Falmer, Brighton, England. 


$$
I(R) \equiv \frac{1}{2 \pi i} \int_{C_{R}}|f(z)|^{2} \frac{d z}{z} \equiv \int_{0}^{1}|f(R \exp (2 \pi i t))|^{2} d t
$$

where $R>r$, and denoting

$$
\rho=r / R
$$

it was shown that

$$
\left|\epsilon_{j, 1}^{(N)}(r)\right|^{2} \leqq I(R) \rho^{2 N+2 j} /\left(1-\rho^{2 N}\right), \quad j=0,1, \cdots, N-1,
$$

and also

$$
\left|r^{j} a_{j}\right|^{2} \leqq I(R) \rho^{2 j} .
$$

In this paper we obtain results of a similar nature corresponding to interpolation and quadrature of analytic functions, for the contours $C$ considered in Paper A: that is, for circles and for squares. The results for circles follow naturally from the results outlined above, and are obtained in the next two sections. The case of a square contour is considered in Section 6.

2. Interpolation for $f(z)$. We suppose that, based on $N$ function evaluations of $f(z)$ at $z=r \exp (2 \pi i j / N), j=1,2, \cdots, N$, we have evaluated approximations $\tilde{a}_{j}^{(N)}$ to $a_{j}, j=0,1, \cdots, N-1$. The value of $f(z)$ at other values of $z$ may be approximated using a power series in $z$. Thus

$$
f(z) \simeq \tilde{f}(z)=\sum_{j=0}^{N-1} \tilde{a}_{j}^{(N)} z^{j} .
$$

The error in this approximation is

$$
f(z)-\tilde{f}(z)=\sum_{j=0}^{N-1}\left(a_{j}-\tilde{a}_{j}^{(N)}\right) z^{j}+\sum_{j=N}^{\infty} a_{j} z^{j} .
$$

We set $\sigma=|z| / r$ and consider values of $z$ satisfying $|z| / R=\rho \sigma<1$. We find

$$
|f(z)-\tilde{f}(z)| \leqq \sum_{j=0}^{N-1}\left|r^{j} a_{j}-r^{j} \tilde{a}_{j}^{(N)}\right| \sigma^{j}+\sum_{j=N}^{\infty}\left|r^{j} a_{j}\right| \sigma^{j} .
$$

The inequalities in Eqs. (1.7) and (1.8) may be substituted here to give

$$
\begin{aligned}
|f(z)-\tilde{f}(z)| & \leqq J(R)\left\{\sum_{j=0}^{N-1} \frac{\rho^{N} \rho^{j}}{\left(1-\rho^{2 N}\right)^{1 / 2}} \sigma^{j}+\sum_{j=N}^{\infty} \rho^{j} \sigma^{j}\right\} \\
& =\frac{\rho^{N}}{1-\rho \sigma}\left\{\frac{1-\rho^{N} \sigma^{N}}{\left(1-\rho^{2 N}\right)^{1 / 2}}+\sigma^{N}\right\} J(R) .
\end{aligned}
$$

In particular, if $z$ lies on or within the circle $|z|=r$

$$
|f(z)-\tilde{f}(z)| \leqq \frac{J(R) \rho^{N}}{1-\rho}\left[\frac{\left(1-\rho^{N}\right)^{1 / 2}}{\left(1+\rho^{N}\right)^{1 / 2}}+1\right] \leqq \frac{2 J(R) \rho^{N}}{1-\rho} .
$$

The algorithms in Paper A involve interpolation to obtain not $f(z)$ but rather $f^{\prime}(z)$ or, more generally, the $\gamma$ th order derivative $f^{(\gamma)}(z)$ where $z$ is on the circle $|z|=r$.

The approximation to $f^{(\gamma)}(z)$ is 


$$
\tilde{f}^{(\gamma)}(z)=\sum_{j=0}^{N-1} \tilde{a}_{j}^{(N)} j(j-1) \cdots(j-\gamma+1) z^{j-\gamma} .
$$

An argument similar to that given above leads to

$$
\begin{array}{r}
\left|r^{\gamma} f^{(\gamma)}(z)-r^{\gamma} \tilde{f}^{(\gamma)}(z)\right| \leqq J(R) \rho^{\gamma}\left\{\frac{\rho^{N}}{\left(1-\rho^{2 N}\right)^{1 / 2}} \sum_{j=0}^{N-1} j(j-1) \cdots(j-\gamma+1) \rho^{j-\gamma}\right. \\
\left.+\sum_{j=N}^{\infty} j(j-1) \cdots(j-\gamma+1) \rho^{j-\gamma}\right\}
\end{array}
$$

where we have assumed $|z|=r$. Since

$$
\sum_{j=0}^{\infty} j(j-1) \cdots(j-\gamma+1) \rho^{j-\gamma}=\frac{\gamma !}{(1-\rho)^{\gamma+1}}
$$

we find

$$
\begin{array}{r}
\left|r^{\gamma} f^{(\gamma)}(z)-r^{\gamma} \tilde{f}^{(\gamma)}(z)\right| \leqq J(R)\left\{\frac{\rho^{\gamma+N} \gamma !}{\left(1-\rho^{2 N}\right)^{1 / 2}(1-\rho)^{\gamma+1}}+\left[1-\frac{\rho^{N}}{\left(1-\rho^{2 N}\right)^{1 / 2}}\right]\right. \\
\left.\times \sum_{j=N}^{\infty} j(j-1) \cdots(j-\gamma+1) \rho^{j}\right\} .
\end{array}
$$

This may be expressed in a form corresponding to (2.5). For example, with $\gamma=1$

$$
\begin{aligned}
\left|r f^{\prime}(z)-r \tilde{f}^{\prime}(z)\right| \leqq \frac{J(R)}{(1-\rho)^{2}}\left\{\rho ^ { N } \left(\frac{\rho}{\left(1-\rho^{2 N}\right)^{1 / 2}}\right.\right. & +N-N \rho+\rho) \\
& \left.+\rho^{2 N}\left(\frac{-N+N \rho-1}{\left(1-\rho^{2 N}\right)^{1 / 2}}\right)\right\} .
\end{aligned}
$$

In general, the asymptotic result is

$$
\left|r^{\gamma} f^{(\gamma)}(z)-r^{\gamma} \tilde{f}^{(\gamma)}(z)\right|<\widetilde{E}_{N}{ }^{(\gamma)}
$$

where

$$
\widetilde{E}_{N}^{(\gamma)} \sim \operatorname{const} N^{\gamma} \rho^{N} \text { as } N \rightarrow \infty .
$$

3. Integration Around a Circle. In this section we are concerned with the evaluation of the contour integral

$$
\frac{1}{2 \pi i} \int_{c} f(z) d z=\int_{0}^{1} r \exp (2 \pi i t) f(r \exp (2 \pi i t)) d t=I \phi(t) .
$$

In this equation, $I \phi(t)$ denotes the integral on the real axis of a periodic function of $t$ over a complete period. In this section we derive an explicit bound on the difference between $I \phi(t)$ and the $N$-point trapezoidal rule approximation to it, namely,

$$
R^{[N, 1]} \phi(t)=\frac{1}{N} \sum_{j=1}^{N} r \exp (2 \pi i j / N) f(r \exp (2 \pi i j / N))
$$

and investigate the asymptotic behavior of this difference. We consider several cases of interest. 
1. $f(z)$ analytic within $|z|=R$. Here $I \phi(t)=0$. If we expand $f(z)$ in a power series we find

$$
R^{[N, 1]} \phi(t)=\frac{1}{N} \sum_{k=0}^{\infty} a_{k} r^{k} \sum_{j=1}^{N} r \exp (2 \pi i j(k+1) / N) .
$$

However

$$
\begin{aligned}
& \sum_{j=1}^{N} \exp (2 \pi i j \gamma / N)=N, \quad \frac{\gamma}{N}=\text { integer } \\
& \sum_{j=1}^{N} \exp (2 \pi i j \gamma / N)=0, \quad \text { otherwise. }
\end{aligned}
$$

Thus

$$
R^{[N, 1]} \phi(t)=r\left(a_{N-1} r^{N-1}+a_{2 N-1} r^{2 N-1}+\cdots\right) .
$$

Using inequality (1.8),

$$
\begin{aligned}
\left|R^{[N, 1]} \phi(t)\right| & \leqq r\left(\left|r^{N-1} a_{N-1}\right|+\left|r^{2 N-1} a_{2 N-1}\right|+\cdots\right) \\
& \leqq r J(R)\left(\rho^{N-1}+\rho^{2 N-1}+\cdots\right)=\frac{r J(R) \rho^{N-1}}{1-\rho^{N}} .
\end{aligned}
$$

Since $I \phi(t)=0$, we conclude that if $f(z)$ is analytic within $|z|=R$, then

$$
\left|R^{[N, 1]} \phi(t)-I \phi(t)\right| \leqq \frac{r J(R) \rho^{N-1}}{1-\rho^{N}} .
$$

2. $f(z)=\left(z-Z_{1}\right)^{-1},\left|Z_{1}\right|<r$. Here we set

$$
\phi(t)=\frac{r \exp (2 \pi i t)}{r \exp (2 \pi i t)-Z_{1}}=\frac{1}{1-\left(Z_{1} / r\right) \exp (-2 \pi i t)} .
$$

It follows from Cauchy's theorem that, since $C_{r}$ contains a simple pole of $f(z)$ of residue 1 ,

$$
\frac{1}{2 \pi i} \int_{C_{r}} f(z) d z=\int_{0}^{1} \phi(t) d t=1
$$

However,

$$
R^{[N, 1]} \phi(t)=\frac{1}{N} \sum_{j=1}^{N} \frac{1}{1-\left(Z_{1} / r\right) \exp (-2 \pi i j / N)} .
$$

Expanding each term as a power series in $\left(Z_{1} / r\right) \exp (-2 \pi i j / N)$ and using (3.4), we find

$$
R^{[N, 1]} \phi(t)=1+\left(Z_{1} / r\right)^{N}+\left(Z_{1} / r\right)^{2 N}+\cdots
$$

Thus

$$
R^{[N, 1]} \phi(t)-I \phi(t)=\left(Z_{1} / r\right)^{N}\left(1-\left(Z_{1} / r\right)^{N}\right)^{-1} .
$$

3. $f(z)=\left(z-Z_{1}\right)^{-1}, r<\left|Z_{1}\right|<R$. Here again 


$$
\phi(t)=\frac{r \exp (2 \pi i t)}{r \exp (2 \pi i t)-Z_{1}}=-\frac{r}{Z_{1}} \exp (2 \pi i t) \frac{1}{1-\left(r / Z_{1}\right) \exp (2 \pi i t)}
$$

but since the pole is outside $C_{r}$

$$
\frac{1}{2 \pi i} \int_{C_{r}} f(z) d z=I \phi(t)=0 .
$$

By a similar argument to case 2

$$
\begin{aligned}
R^{[N, 1]} \phi(t) & =-\frac{1}{N} \sum_{j=1}^{N} \frac{r}{Z_{1}} \exp (2 \pi i j / N)\left(1-\frac{r}{Z_{1}} \exp (2 \pi i j / N)\right)^{-1} \\
& =-\left(\frac{r}{Z_{1}}\right)^{N}-\left(\frac{r}{Z_{1}}\right)^{2 N}-\cdots
\end{aligned}
$$

Thus

$$
R^{[N, 1]} \phi(t)-I \phi(t)=-\left(r / Z_{1}\right)^{N}\left(1-\left(r / Z_{1}\right)^{N}\right)^{-1} .
$$

4. $f(z)=\psi^{\prime}(z) z^{\gamma} / \psi(z)$ where $\gamma$ is an integer $\geqq 0$ and $\psi(z)$ is analytic within $|z|=R$. This is the case needed in Paper A. Suppose that $\psi(z)$ has zeros within $|z|=r$ at $z=Z_{1}, Z_{2}, \cdots, Z_{\nu}$ and has zeros within $r<|z|<R$ at $z=Z_{\nu+1}, Z_{\nu+2}, \cdots, Z_{d}$ (multiple zeros being counted according to their multiplicity). The partial fraction expansion of $f(z)$ is

$$
f(z)=\sum_{i=1}^{\nu} \frac{Z_{i}^{\gamma}}{z-Z_{i}}+\sum_{i=\nu+1}^{d} \frac{Z_{i}{ }^{\gamma}}{z-Z_{i}}+\tilde{f}(z)
$$

where $\tilde{f}(z)$ is analytic within $|z|=r$. Since $R^{[N, 1]}-I$ is a linear functional, and $f(z)$ is the sum of cases considered previously, we may combine the results in these cases to find

$$
\begin{aligned}
& \left|\frac{1}{N} \sum_{j=1}^{N} r \exp (2 \pi i j / N) f(r \exp (2 \pi i j / N))-\frac{1}{2 \pi i} \int_{C} f(z) d z\right| \\
& \leqq \\
& \quad+\frac{\sum_{i=1}^{\nu} Z_{i}^{\gamma}\left(\frac{Z_{i}}{r}\right)^{N}\left(1-\left(\frac{Z_{i}}{r}\right)^{N}\right)^{-1}+\sum_{i=\nu+1}^{d} Z_{i}^{\gamma}\left(\frac{r}{Z_{i}}\right)^{N}\left(1-\left(\frac{r}{Z_{i}}\right)^{N}\right)^{-1} \mid}{\rho\left(1-\rho^{N}\right)},
\end{aligned}
$$

where $\tilde{J}(R)$ is given by (1.5) above but with $\tilde{f}(z)$ replacing $f(z)$. This equation gives an upper bound on the asymptotic behavior of the discretization error for large $N$. We may express this

$$
\left|R^{[N, 1]} \phi(t)-I \phi(t)\right| \leqq G(N) \sim \operatorname{const} A^{N} \text { as } N \rightarrow \infty
$$

where $|A|<1$ and is given by

$$
|A|=\max \left(A_{1}, A_{2}, A_{3}\right)
$$

where

$$
\begin{gathered}
A_{1}=\max \left|Z_{i} / r\right|, \quad\left|Z_{i}\right|<r, \quad A_{2}=\max \left|r / Z_{i}\right|, \quad\left|Z_{i}\right|>r, \\
A_{3}=r / R .
\end{gathered}
$$


Eq. (3.19) indicates that the error may be considered to be linear in the number of function evaluations; that is, asymptotically each additional function evaluation reduces the error by the constant factor $A$.

4. Romberg Integration Adapted to Particular Expansions. In Section 6 we deal with the application of Romberg integration to contour integration round a square contour. This application requires a particular modification of the standard technique. In this section and in Section 5 we derive results of a theoretical nature which relate to integration along lines. These are required in Section 6.

The standard treatment of Romberg integration is given in Bauer, Rutishauser and Stiefel [1]. We refer to this paper as B.R.S. We employ in this section the notation

$$
\begin{aligned}
R^{[N, 1]} f \equiv \frac{1}{N} \sum_{j=0}^{N} f^{\prime \prime}\left(\frac{j}{N}\right) \equiv \frac{1}{N}\left\{\frac{1}{2} f(0)+f\left(\frac{1}{N}\right)+\right. & f\left(\frac{2}{N}\right) \\
& \left.+\cdots+f\left(\frac{N-1}{N}\right)+\frac{1}{2} f(1)\right\}
\end{aligned}
$$

the primes on the summation indicating that the first and last terms are assigned a weighting factor $\frac{1}{2}$. The treatment may be based on the Euler-Maclaurin expansion

$$
\begin{aligned}
R^{[m, 1]} f-I f= & \frac{c_{2}}{m^{2}}\left(f^{\prime}(1)-f^{\prime}(0)\right)+\frac{c_{4}}{m^{4}}\left(f^{\prime \prime \prime}(1)-f^{\prime \prime \prime}(0)\right) \\
& +\cdots+\frac{c_{2 p-2}}{m^{2 p-2}}\left(f^{(2 p-3)}(1)-f^{(2 p-3)}(0)\right) \\
& +\frac{-2(-1)^{p}}{(2 \pi m)^{2 p}} \int_{0}^{1} f^{(2 p)}(x) \sum_{r=1}^{\infty} \frac{1-\cos 2 \pi r m x}{r^{2 p}} d x
\end{aligned}
$$

where

$$
c_{2 s}=(-1)^{s-1} 2 \zeta(2 s) /(2 \pi)^{2 s}=(-1)^{s-1}\left|B_{2 s}\right| /(2 s) ! .
$$

In the normal Romberg treatment (B.R.S.), $R^{[m, 1]} f$ is evaluated for mesh ratios $m=1,2,4,8, \cdots$ and the leading terms in the $T$-table are

$$
T_{0}{ }^{(k)}=R^{\left[m_{k}, 1\right]} f, \quad m_{k}=2^{k}, \quad k=0,1, \cdots .
$$

The conventional $T$-table is constructed using the recurrence relation

$$
T_{s}^{(k)}=T_{s-1}^{(k+1)}+\frac{T_{s-1}^{(k+1)}-T_{s-1}^{(k)}}{4^{s}-1} .
$$

$T_{s}{ }^{(k)}$ is a linear combination $T_{0}{ }^{(k)}, T_{0}{ }^{(k+1)}, \cdots, T_{0}{ }^{(k+s)}$ and is precisely that combination which eliminates the coefficients of $m^{2}, m^{4}, \cdots, m^{2 s}$ in expansion (4.1) above. B.R.S. shows that

$$
T_{s}{ }^{(k)}-I f=4^{-(s+1) k} \int_{0}^{1} b_{2 s+2}\left(2^{k} x\right) f^{(2 s+2)}(x) d x
$$

where $b_{2 s+2}(x)$ is positive definite and 


$$
\int_{0}^{1} b_{2 s+2}(x) d x=\frac{\left|B_{2 s+2}\right|}{(2 s+2) ! 2^{s(s+1)}} .
$$

In the conventional notation

$$
h=1 / m
$$

is referred to as the step size or as the discretization parameter. The standard theory, described above, refers to the usual case in which the discretization error has what is known as an $h^{2}$ expansion, that is, of the form

$$
E(h) \sim c_{2} h^{2}+c_{4} h^{4}+c_{6} h^{6}+\cdots
$$

where

$$
c_{2 n} \neq 0, \quad n=1,2, \cdots .
$$

In the case of contour integration round a square (see Section 6 ) the final result is independent of the values of $c_{4}, c_{8}, \cdots$, and the calculation of $E(h)$ for a given side can therefore be carried out as if

$$
E(h) \sim c_{2} h^{2}+c_{6} h^{6}+c_{10} h^{10}+\cdots .
$$

Other types of discretization errors occur which involve some but not all even powers of $h$. For example, in contour integration round triangles or hexagons, the effective discretization error is

$$
E(h) \sim c_{2} h^{2}+c_{4} h^{4}+c_{8} h^{8}+c_{10} h^{10}+c_{14} h^{14}+\cdots,
$$

while in two-dimensional quadrature over a square of a harmonic function

$$
E(h) \sim c_{4} h^{4}+c_{8} h^{8}+c_{12} h^{12}+\cdots .
$$

In this section we do not restrict ourselves specifically to (4.8), since the princioal result is independent of precisely which terms are included and which are omitted.

We may construct an adaption of the $T$-table to suit the case under consideration. If a particular term $\left(c_{2 s} / m^{2 s}\right)\left(f^{(2 s-1)}(1)-f^{(2 s-1)}(0)\right)$ is not to be eliminated, we set

$$
T_{s}^{(k)}=T_{s-1}^{(k)} .
$$

Otherwise we use the standard relation (4.3). Thus at any stage, the relation which gives $T_{s}{ }^{(k)}$ in terms of elements of the previous column is either

$$
T_{s}^{\left({ }^{(k)}\right.}=T_{s-1}^{(k)} \text { (noneliminating step) }
$$

or

$$
T_{s}^{(k)}=T_{s-1}^{(k+1)}+\frac{T_{s-1}^{(k+1)}-T_{s-1}^{(k)}}{4^{s}-1} \text { (eliminating step) } .
$$

The structure of the $T$-table differs from the normal structure. For example, in the case in which the terms in $m^{4}, m^{8}$, and $m^{12}$ are not to be eliminated, the table has the appearance 


$$
\begin{array}{ll}
T_{0}{ }^{(0)} & \searrow T_{1}{ }^{(0)}=T_{2}{ }^{(0)} \searrow T_{3}{ }^{(0)}=T_{4}{ }^{(0)} \\
T_{0}{ }^{(1)} & \left.\searrow T_{1}{ }^{(1)}=T_{2}{ }^{(1)} \searrow T_{3}{ }^{(1)}=T_{4}{ }^{(1)}\right\rangle T_{5}{ }^{(0)}=T_{6}{ }^{(0)} \\
T_{0}{ }^{(3)} & \searrow T_{1}{ }^{(2)}=T_{2}{ }^{(2)}
\end{array}
$$

Figure 1

We now establish the following

THEOREM.

$$
\begin{aligned}
T_{m}{ }^{(k)}-I f= & \int_{0}^{1} \phi(x) f^{(2 m+2)}(x) d x \\
& +\sum_{j=1}^{m} A_{m, j}^{(k)}\left(f^{(2 j-1)}(1)-f^{(2 j-1)}(0)\right)
\end{aligned}
$$

where $\phi(x)$ is of definite sign, and $A_{m, j}^{(k)}$ are certain numbers defined below.

It is convenient to define two classes of functions, $E$ (even) and $O$ (odd).

Definition. A function $\phi(x)$ is of class $E$ if

(i) $\phi(x)$ is of definite sign $0<x<1$,

(ii) $\phi^{\prime}(x)$ is of definite sign $0<x<\frac{1}{2}$,

(iii) $\phi(0)=\phi(1)=0$,

(iv) $\phi(x)=\phi(1-x)$,

(v) $\phi(x)=\phi(x+1)$.

For example $\phi(x)=|\sin \pi x|$ is of class $E$.

Definition. A function $\phi(x)$ is of class $O$ if

(i) $\phi(x)$ is of definite sign, $0<x<\frac{1}{2}$,

(ii) $\phi(0)=\phi\left(\frac{1}{2}\right)=\phi(1)$,

(iii) $\phi(x)=-\phi(1-x)$,

(iv) $\phi(x)=\phi(x+1)$.

For example $\phi(x)=\sin 2 \pi x$ is of class $O$.

We state without proof three simple lemmas connecting functions of these classes.

Lemma 1. If $\phi(x)$ is of class $O$, then

$$
\psi(x)=\int_{0}^{x} \phi(x) d x
$$

is of class $E$.

LEMma 2. If $\phi(x)$ is of class $E$

$$
\psi(x)=\int_{0}^{x}(\phi(2 x)-\phi(x)) d x
$$

is of class $O$.

Lemma 3. If $\phi(x)$ is of class $E$

$$
\psi(x)=\int_{0}^{x} \phi(x) d x-x \int_{0}^{1} \phi(x) d x
$$

is of class $O$. 
It is shown in B.R.S. that

$$
T_{0}{ }^{(k)}-I f=4^{-k} \int_{0}^{1} b_{2}\left(2^{k} x\right) f^{\prime \prime}(x) d x
$$

where

$$
\begin{aligned}
b_{2}(x) & =\frac{1}{2} x(1-x), & & 0 \leqq x \leqq 1, \\
b_{2}(1+x) & =b_{2}(x), & & \text { all } x .
\end{aligned}
$$

It is clear that $b_{2}(x)$ is a function of class $E$.

We establish the theorem by induction. The theorem is clearly true for $s=1$. We assume that it is true for $s=m-1, m-2, \cdots, 1$. Thus

$$
\begin{aligned}
T_{m-1}^{(k)}-I f= & 4^{-m k} \int_{0}^{1} b_{2 m}\left(2^{k} x\right) f^{(2 m)}(x) d x \\
& +\sum_{j=1}^{m-1} A_{m-1, j}^{(k)}\left(f^{(2 j-1)}(1)-f^{(2 j-1)}(0)\right)
\end{aligned}
$$

where $b_{2 m}(x)$ is of class $E$.

There are two cases to consider. These are (i) if the step from $T_{m-1}^{(k)}$ to $T_{m}{ }^{(k)}$ is an eliminating step (4.13) and (ii) if this step is a noneliminating step (4.12).

In case (i) we simply follow B.R.S. We define

$$
\begin{aligned}
& b_{2 m+1}(x)=\int_{0}^{x}\left(b_{2 m}(2 x)-b_{2 m}(x)\right) d x /\left(4^{m}-1\right), \\
& b_{2 m+2}(x)=\int_{0}^{x} b_{2 m+1}(x) .
\end{aligned}
$$

Since $b_{2 m}(x)$ is of class $E$, Lemmas 1 and 3 indicate that $b_{2 m+2}(x)$ is also of class $E$. Then we calculate $T_{m}{ }^{(k)}-I f$ from (4.13) and (4.17). We find directly

$$
\begin{aligned}
T_{m}{ }^{(k)}-I f= & \frac{4^{-k m}}{4^{m}-1} \int_{0}^{1}\left(b_{2 m}\left(2^{k+1} x\right)-b_{2 m}\left(2^{k} x\right)\right) f^{(2 m)}(x) d x \\
& +\sum_{j=1}^{m-1} \frac{4^{m} A_{m-1, j}^{(k+1)}-A_{m-1, j}^{(k)}}{4^{m}-1}\left(f^{(2 j-1)}(1)-f^{(2 j-1)}(0)\right) .
\end{aligned}
$$

We may carry out an integration by parts twice and set

$$
\begin{gathered}
A_{m, m}^{(k)}=0, \\
A_{m, j}^{(k)}=\left(4^{m} A_{m-1, j}^{(k+1)}-A_{m-1, j}^{(k)}\right) /\left(4^{m}-1\right), \quad j=1,2, \cdots, m-1 .
\end{gathered}
$$

This puts (4.20) in the same form as (4.17) but with $m-1$ replaced by $m$.

In case (ii) we proceed in an analogous but different way. We define

$$
\begin{aligned}
\int_{0}^{1} b_{2 m}\left(2^{k} x\right) d x & =K_{2 m} \\
\hat{b}_{2 m}(x) & =b_{2 m}(x)-K_{2 m}, \quad 0 \leqq x \leqq 1, \\
b_{2 m+1}(x) & =\int_{0}^{x} \hat{b}_{2 m}(x) d x,
\end{aligned}
$$




$$
b_{2 m+2}(x)=\int_{0}^{x} b_{2 m+1}(x) d x .
$$

Since $b_{2 m}(x)$ is of class $E$, Lemmas 1 and 2 show that $b_{2 m+2}(x)$ is also of class $E$.

Using (4.12) and (4.17) it follows that

$$
\begin{aligned}
T_{m}{ }^{(k)}-I f & =4^{-m k} \int_{0}^{1}\left(b_{2 m}\left(2^{k} x\right)-K_{2 m}\right) f^{(2 m)}(x) d x \\
& +4^{-m k} K_{2 m} \int_{0}^{1} f^{(2 m)}(x) d x+\sum_{j=1}^{m-1} A_{m-1, j}^{(k)}\left(f^{(2 j-1)}(1)-f^{(2 j-1)}(0)\right) .
\end{aligned}
$$

The second term may be written

$$
A_{m, m}^{(k)}\left(f^{(2 m-1)}(1)-f^{(2 m-1)}(0)\right)
$$

where

$$
A_{m, m}^{(k)}=4^{-m k} K_{2 m}
$$

We also define

$$
A_{m, j}^{(k)}=A_{m-1, j}^{(k)}, \quad j=1,2, \cdots, m-1 .
$$

The integrand in the first term is $\hat{b}_{2 m}(x)$. Integration by parts twice leads to

$$
\begin{aligned}
T_{m}{ }^{(k)}-I f= & 4^{-(m+1) k} \int_{0}^{1} b_{2 m+2}\left(2^{k} x\right) f^{(2 m+2)}(x) d x \\
& +\sum_{j=1}^{m} A_{m, j}^{(k)}\left(f^{(2 j-1)}(1)-f^{(2 j-1)}(0)\right) .
\end{aligned}
$$

This is of exactly the same form as (4.17) with $m-1$ replaced by $m$.

Thus in both cases (i) and (ii), corresponding to the step being eliminating or noneliminating, the form of (4.17) is retained after the next step. Moreover, since in each case $b_{2 m+2}(x)$ is of class $E, b_{2 m+2}\left(2^{k} x\right)$ is of definite sign. This establishes the theorem (4.14).

The above proof constitutes rather a lengthy proof of the Euler-Maclaurin summation formula if we choose all the steps to be noneliminating. On the other hand if all the steps are eliminating, it reduces to the standard treatment of B.R.S.

Theorem (4.14) has the immediate corollary obtained through the intermediatevalue theorem:

Corollary.

$$
T_{m}{ }^{(k)}-I f=C_{m}{ }^{(k)} f^{(2 m+2)}(\xi)+\sum_{j=1}^{m} A_{m, j}^{(k)}\left(f^{(2 j-1)}(1)-f^{(2 j-1)}(0)\right)
$$

where $0 \leqq \xi \leqq 1$ and

$$
C_{m}{ }^{(k)}=4^{-(m+1) k} \int_{0}^{1} b_{2 m+2}(x) d x .
$$

We note that the numbers $A_{m, j}^{(k)}$ are generated according to one or another of the several relations (4.21), (4.22), (4.29) and (4.30). However, if the $n$th step is an eliminating step, i.e., 


$$
T_{n}{ }^{(k)}=T_{n-1}^{(k+1)}+\frac{T_{n-1}^{(k+1)}-T_{n-1}^{(k)}}{4^{n}-1}
$$

it follows from (4.21) that

$$
A_{n, n}^{(k)}=0 .
$$

However, $A_{m, n}^{(k)}$ is obtained by one of

$$
A_{m, n}^{(k)}=\left(4^{m} A_{m-1, n}^{(k+1)}-A_{m-1, n}^{(k)}\right) /\left(4^{m}-1\right)
$$

or

$$
A_{m, n}^{(k)}=A_{m-1, n}^{(k)} .
$$

Thus, in either case, letting $m=n+1, n+2, \cdots$, in turn we get

$$
A_{m, n}^{(k)}=0 \quad \text { all } m \geqq n .
$$

This simply confirms that the procedure does in fact eliminate those terms in the Euler-Maclaurin series which it is designed to do.

We state this as a corollary:

$$
A_{m, n}^{(k)}=0 \quad \text { if the } n \text {th step is an eliminating step. }
$$

5. A Bound on the Truncation Error in a Particular Case. We now restrict ourselves to the particular sequences in which we eliminate terms in $h^{2}, h^{6}, h^{10}, \cdots$. That is, we proceed as if

$$
E(h) \sim c_{2} h^{2}+c_{6} h^{6}+c_{10} h^{10}+\cdots .
$$

This is appropriate in the case of contour integration round squares. The $T$-table is then exactly as illustrated in Fig. 1 the odd-numbered steps being eliminating steps and the even-numbered steps being noneliminating steps. Thus

$$
A_{m, j}^{(k)}=0, \quad j \text { odd . }
$$

It is clear from the construction of the $T$-table that $T_{2 p}^{(0)}$ is a well-defined linear combination of $T_{0}{ }^{(j)}, j=0,1, \cdots, p$. Thus

$$
T_{2 p}^{(0)}=\gamma_{0} T_{0}{ }^{(0)}+\gamma_{1} T_{0}{ }^{(1)}+\cdots+\gamma_{p} T_{0}{ }^{(p)} .
$$

Since

$$
T_{0}{ }^{(k)}=R^{\left[2^{k}, 1\right]} f
$$

the Euler-Maclaurin summation formula takes the form

$$
T_{0}{ }^{(k)}-I f=\sum_{j=1}^{2 p} \frac{c_{2 j}}{4^{k j}}\left(f^{(2 j-1)}(1)-f^{(2 j-1)}(0)\right)+\int_{0}^{1} f^{(4 p+2)}(x) \phi(x) d x
$$

where $\phi(x)$ is bounded. Consequently, using (5.3),

$$
\begin{aligned}
T_{2 p}^{(0)}-I f \sum_{k=0}^{p} \gamma_{k}= & \sum_{j=1}^{2 p} c_{2 j} \sum_{k=0}^{p} \frac{\gamma_{k}}{4^{k j}}\left(f^{(2 j-1)}(1)-f^{(2 j-1)}(0)\right) \\
& +\int_{0}^{1} f^{(4 p+2)}(x) \tilde{\phi}(x) d x
\end{aligned}
$$


where $\tilde{\phi}(x)$ is bounded. However, according to the theorem

$$
T_{2 p}^{(0)}-I f=\sum_{j=1}^{2 p} A_{2 p, j}^{(0)}\left(f^{(2 j-1)}(1)-f^{(2 j-1)}(0)\right)+C_{2 p}^{(0)} f^{(2 p+2)}(\xi) .
$$

These two expressions for the same discretization error may be used to identify corresponding terms. By successively choosing $f(x)$ to be a polynomial of degree $0,2,4,6, \cdots$, we find

$$
\begin{aligned}
\sum_{k=0}^{p} \gamma_{k} & =1, \\
\sum_{k=0}^{p} \gamma_{k} / 4^{k j} & =0, \quad j=1,3, \cdots, 2 p-1, \\
\sum_{k=0}^{p} \gamma_{k} / 4^{k j} & =A_{2 p, j}^{(0)} / c_{2 j}, \quad \jmath=2,4, \cdots, 2 p, \\
C_{2 p}^{(0)} & =c_{4 p+2} \sum_{k=0}^{p} \gamma_{k} / 4^{(2 p+1) k} .
\end{aligned}
$$

The summation in (5.11) is algebraic in character. The $p+1$ equations (5.8) and (5.9) together define $\gamma_{k}(k=0,1, \cdots, p)$. The sum in (5.11) may be expressed in the form of a quotient of two determinants of the Vandermonde type containing many common factors. The result is

$$
\sum_{k=0}^{p} \gamma_{k} / 4^{(2 p+1) k}=4^{-\left(p^{2}\right)} \frac{\left(1-2^{-4}\right)\left(1-2^{-8}\right) \cdots\left(1-2^{-4 p}\right)}{\left(1-2^{-2}\right)\left(1-2^{-6}\right) \cdots\left(1-2^{-(4 p-2)}\right)} .
$$

The factor of $4^{-\left(p^{2}\right)}$ in this expression is a number lying between $1 \frac{1}{4}$ and $1 \frac{1}{3}$. Since according to (4.1a)

$$
c_{4 p+2}=\frac{2 \zeta(4 p+2)}{(2 \pi)^{4 p+2}}
$$

and

$$
1<\zeta(4 p+2) \leqq \zeta(6)<1.1,
$$

we may combine (4.33), (5.11), (5.12), (5.13), and (5.14) to give the following result

$$
\begin{aligned}
C_{2 p}^{(k)} & =4^{-(2 p+1) k} C_{2 p}^{(0)} \\
& =4^{-(2 p+1) k} \frac{2 \zeta(4 p+2)}{(2 \pi)^{4 p+2}} 4^{-\left(p^{2}\right)} \prod_{j=1}^{p}\left(1-4^{-2 j}\right)\left(1-4^{-2 j+1}\right)^{-1}
\end{aligned}
$$

or in a more useful form

$$
\left|C_{2 p}^{(k)}\right|=4^{-(2 p+1) k}\left|C_{2 p}^{(0)}\right|<4^{-(2 p+1) k} \frac{3 \cdot 4^{-\left(p^{2}\right)}}{(2 \pi)^{4 p+2}} .
$$

This completes the determination of $C_{2 p}^{(k)}$. We complete the estimation of the truncation error $C_{2 p}^{(k)} f^{(4 p+2)}(\xi)$ in the case in which $f(x)$ is a complex analytic function in a region containing the interval of integration $[0,1]$. In this case, if $\rho$ is the shortest distance from this interval to any singularity of $f(z)$, it follows that for any 
fixed $\epsilon>0$ there exists a number $K(\epsilon)$ such that

$$
\left|f^{(p)}(x) / p !\right|<K(\epsilon) /(\rho-\epsilon)^{p} ;
$$

consequently

$$
\left|C_{2 p}^{(k)} f^{(4 p+2)}(\xi)\right|<\frac{3 K(\epsilon) 4^{-(2 p+1) k} 4^{-\left(p^{2}\right)}(4 p+2) !}{(2 \pi)^{4 p+2}(\rho-\epsilon)^{4 p+2}} .
$$

In the case of a simple pole being the closest singularity we may take $\epsilon=0$.

6. A Square Contour. In this section we consider integration round a square contour. The results of Sections 4 and 5 are required in this section to bound the discretization error.

We consider first the use of the trapezoidal rule with (complex) step length $h=(b-a) / m$ to carry out contour integration from $a$ to $b$ along a straight line in the complex plane. Since $f(z)$ is an analytic function we may apply the EulerMaclaurin summation formula

$$
\begin{aligned}
h \sum_{j=0}^{m} \prime \prime f(a+h j)-\int_{a}^{b} f(z) d z= & \sum_{r=1}^{2 p} c_{2 r} h^{2 r}\left\{f^{(2 r-1)}(b)-f^{(2 r-1)}(a)\right\} \\
& +\int_{a}^{b} \phi(z) f^{(2 p+2)}(z) d z
\end{aligned}
$$

where $\phi(z)$ is bounded on the line $a b$.

We now consider the closed polygonal contour whose vertices in order are the complex numbers $a_{1}, a_{2}, \cdots, a_{n}$. We suppose that the trapezoidal rule with step length

$$
h_{j}=\left(a_{j+1}-a_{j}\right) / m_{j}
$$

is used for the section between $a_{j}$ and $a_{j+1}$. Applying (6.1) we find

$$
\begin{gathered}
\sum_{k=1}^{N} h_{k} \sum_{j=0}^{m_{k}} f\left(a_{k}+j h_{k}\right)-\int_{c\left(a_{1}, a_{2}, \ldots, a_{n}\right)} f(z) d z \\
=\sum_{r=1}^{2 p} c_{2 r} \sum_{k=1}^{N} f^{(2 r-1)}\left(a_{k}\right)\left\{h_{k-1}^{2 r}-h_{k}^{2 r}\right\} \\
\quad+\int_{c} \tilde{\phi}_{p}(z) f^{(4 p+2)}(z) d z
\end{gathered}
$$

where $\tilde{\phi}_{p}(z)$ is bounded. The particular case in which we are interested is that in which $a_{1}, a_{2}, a_{3}$, and $a_{4}$ are the vertices of a square of side $2 R$, and the same step size is used on each side. That is, we set

$$
h_{1}=-i h_{2}=-h_{3}=i h_{4}=2 R e^{i \phi} / m
$$

where $\phi$ is the angle between the real axis and the first side. With this choice of step size,

$$
h_{k-1}^{4 r}=h_{k}^{4 r}, \quad r=\text { integer },
$$

hence the terms in (6.3) with $r$ even are zero and 


$$
\begin{aligned}
\sum_{k=1}^{4} h_{k} \sum_{j=0}^{m} \prime \prime f\left(a_{k}\right. & \left.+j h_{k}\right)-\int_{\square} f(z) d z \\
= & \sum_{r=1}^{p} 2 c_{4 r-2} \exp (-2 i(2 r-1) \phi)\left(\frac{2 R}{m}\right)^{4 r-2} \\
& \times\left\{f^{4 r-3)}\left(a_{2}\right)+f^{(4 r-3)}\left(a_{4}\right)-f^{(4 r-3)}\left(a_{1}\right)-f^{(4 r-3)}\left(a_{3}\right)\right\} \\
& +\int_{\square} \tilde{\phi}_{p}(z) f^{(4 p+2)}(z) d z .
\end{aligned}
$$

This asymptotic expansion for the discretization error contains terms in $m^{-2}, m^{-6}$, $m^{-10}, \cdots$, but not in $m^{-4}, m^{-8}, m^{-12}, \cdots$; that is, it is of the type (4.8). We use trapezoidal sums based on halving the mesh ratio at each step. Thus we set

$$
m_{i}=2^{i}
$$

and define

$$
S_{0}^{(i)}=\sum_{k=1}^{4} \frac{a_{k+1}-a_{k}}{m_{i}} \sum_{j=0}^{m_{i}}{ }^{\prime \prime} f\left(a_{k}+j\left(\frac{a_{k+1}-a_{k}}{m_{i}}\right)\right) .
$$

The modification of the standard Romberg $T$-table, described in Sections 4 and 5 may now be constructed. We obtain in this way a linear combination of $S_{0}^{(i)}$, $i=0,1, \cdots, p$

$$
S_{2 p}^{(0)}=\gamma_{0} S_{0}{ }^{(0)}+\gamma_{1} S_{0}^{(1)}+\cdots+\gamma_{p} S_{0}^{(p)}
$$

where the $\gamma_{i}$ are defined by (5.8) and (5.9). Eq. (6.6) may now be written in the form

$$
S_{0}{ }^{(i)}-\int_{\square} f(z) d z=\sum_{r=1}^{p} \frac{d_{r}}{4^{(2 r-1 i)}}+\int_{\square} \tilde{\phi}_{p}(z) f^{(4 p+2)}(z) d z
$$

and it follows directly using (6.9), (5.8) and (5.9) that

$$
S_{2 p}^{(0)}-\int_{\square} f(z) d z=\int_{\square} \Phi(z) f^{(4 p+2)}(z) d z
$$

where

$$
\Phi(z)=\angle \gamma_{i} \tilde{\phi}_{m_{i}}(z)
$$

is bounded on the sides of the square.

We now obtain a bound on this remainder term. Considering for the moment the first of these sides $a_{1} a_{2}$, a simple change of variable,

$$
\begin{aligned}
z & =a_{1}+\left(a_{2}-a_{1}\right) t, \\
f(z) & =F_{1}(t),
\end{aligned}
$$

gives

$$
\begin{aligned}
h_{1} \sum_{j=0}^{m} \prime \prime f\left(a_{1}\right. & \left.+h_{1} j\right)-\int_{a_{1}}^{a_{2}} f(z) d z \\
& =\left(a_{2}-a_{1}\right)\left\{\frac{1}{m} \sum_{j=0}^{m \prime \prime} F_{1}\left(\frac{j}{m}\right)-\int_{0}^{1} F_{1}(t) d t\right\} .
\end{aligned}
$$


Thus $S_{0}^{(i)}$ is the sum of four terms, one of which is given in (6.15) with $m=2^{i}$. Consequently,

$$
S_{2 p}^{(0)}-\int_{\square} f(z) d z=\sum_{i=0}^{p} \gamma_{i} S_{0}{ }^{(i)}-\int_{\square} f(z) d z
$$

may be expressed as the sum of four terms, one of which we denote by $\Lambda_{1}$ :

$$
\Lambda_{1}=\left(a_{2}-a_{1}\right) \sum_{i=0}^{p} \gamma_{i}\left(\frac{1}{m_{i}} \sum_{j=0}^{m_{i}} F_{1}\left(\frac{j}{m}\right)-\int_{0}^{1} F_{1}(t) d t\right) .
$$

Sections 4 and 5 were devoted to obtaining a bound on an expression of this type. There it was shown that

$$
\Lambda_{1}=\left(a_{2}-a_{1}\right) \sum_{j=1}^{p} A_{2 p, 2 j}^{(0)}\left(F_{1}{ }^{(4 j-1)}(1)+F_{1}{ }^{(4 j-1)}(0)\right)+\left(a_{2}-a_{1}\right) E_{1}
$$

where

$$
E_{1}=C_{2 p}^{(0)} F_{1}^{(4 p+2)}\left(\xi_{1}\right)
$$

and is bounded by

$$
\left|E_{1}\right|<\frac{(4 p+2) ! 3 K_{1}(\epsilon)}{4^{\left(p^{2}\right)}\left(2 n\left(\rho_{1}-\epsilon\right)\right)^{4 p+2}} .
$$

Here the suffix 1 indicates that the quantities refer to the side $a_{1} a_{2}$ and we have used (5.2), namely

$$
A_{m, j}^{(0)}=0, \quad j \text { odd } .
$$

To obtain a bound for $S_{2 p}^{(0)}$ we add to (6.18) the similar expressions corresponding to the other three sides. In virtue of $(6.12)$ there are no terms in $f^{(4 j-1)}(1)$ $f^{(4 j-1)}(0)$ in the result. Consequently, the first set of terms on the right-hand side of (6.18) combine with similar terms from the other three sides to give zero. Thus

$$
\begin{aligned}
S_{2 p}^{(0)}-\int_{\square} f(z) d z= & \left(a_{2}-a_{1}\right) E_{1}+\left(a_{3}-a_{2}\right) E_{2} \\
& +\left(a_{4}-a_{3}\right) E_{3}+\left(a_{1}-a_{4}\right) E_{4} .
\end{aligned}
$$

Since $f(z)$ is an analytic function, so is $F_{1}(t)$. The quantity $\rho_{1}$ is the shortest distance from a singularity of $F_{1}(t)$ to the interval $[0,1]$ and $2 R \rho_{1}$ is the corresponding distance in the $z$ plane. Thus we set

$$
d=2 R \min \rho_{j}
$$

and

$$
K(\epsilon)=4 \max K_{j}(\epsilon)
$$

We find

$$
\left|S_{2 p}^{(0)}-\int_{\square} f(z) d z\right| \leqq \frac{(4 p+2) ! 3 K(\epsilon)}{4^{\left(p^{2}\right)}(2 \pi)^{4 p+2}(d / 2 R-\epsilon)^{4 p+2}} .
$$

The case of interest in Paper A is 


$$
f(z)=z^{N} \psi^{\prime}(z) / \psi(z)
$$

where $\psi(z)$ is an analytic function. In this case $f(z)$ has only simple poles and we may set $\epsilon=0$ in (6.24) above.

In terms of the number of iterations the convergence rate indicated by (6.24) is superlinear. However, each iteration doubles the number of function evaluations, $N$. If we express $E$ in terms of $N$, we find

$$
E(N) \sim \text { const } \frac{1}{N^{2 \log _{2} N}} \cdot \frac{\left(\log _{2} N\right)^{\left(4 \log _{2} N-5 \cdot 5\right)}}{N^{4\left(\log _{2}(n \rho e \cdot 4)\right)}} \text { as } N \rightarrow \infty .
$$

For large $N$ the dominant term in this expression is $1 / N^{2 \log _{2} N}$. The ratio of the error in two successive iterations is given by

$$
\frac{E(2 N)}{E(N)} \sim \frac{2^{6}}{\pi^{4}} \frac{\left(\log _{2} N\right)^{2}}{N^{4}} \text { as } N \rightarrow \infty .
$$

The convergence rate is slower than the linear rate exhibited by circles (see (3.19)). There

$$
E(N) \sim \operatorname{const} A^{N}, \quad|A|<1 \text { as } N \rightarrow \infty
$$

and

$$
\frac{E(2 N)}{E(N)} \sim A^{N} \text { as } \quad N \rightarrow \infty .
$$

7. Discussion. Contour integration round a closed contour is a discipline refreshingly free from many of the annoying restrictions encountered in the more familiar field of one-dimensional integration. There, if the region of integration passes near a singularity of the integrand, one must live with this fact. To evaluate a contour integral, one is usually free to adjust the contour to avoid passing near these singularities.

This paper represents only a first short step into a wide field. Two of the simplest contours have been treated, and then only the obvious quadrature rules have been applied. There remains considerable scope for further investigation.

However, even this investigation provides an insight into the question of altering the contour. Perhaps the most striking difference between conventional quadrature and contour integration is this. In both of the cases considered here, each iteration involves doubling the number of function evaluations. At the end of $m$ iterations, $2^{m}$ function evaluations have been made. The next iteration requires a further $2^{m}$ function evaluation. The user may prefer to abandon the particular contour for an apparently more favorable one. If he does this he may carry out $m$ iterations on the new contour at the same cost as the $(m+1)$ th iteration on the original contour, the cost being measured in terms of function evaluations.

We look at this in more detail in the case of a circular contour. We suppose that the original contour 1 has a nearest singularity (or pole) at a distance $\rho_{1}$, and that for the alternative contour 2 , the distance to the nearest singularity is $\rho_{2}$; in both cases we measure distances in terms of the radius of the contour. We denote by 
$E_{1}(N)$ the error after $N$ function evaluations using contour 1 and $E_{2}(N)$ similarly with respect to contour 2 . If we already have a result based on $N$ function evaluations using contour 1, the error after a total of $2 N$ evaluations is either $E_{1}(2 N)$ or $E_{2}(N)$, depending on whether we carry on with contour 1 or we change to contour 2 .

A trivial calculation based on (3.19) shows that

$$
\frac{E_{1}(2 N)}{E_{2}(N)} \sim \operatorname{const}\left(\frac{A_{1}{ }^{2}}{A_{2}}\right)^{N} \sim \operatorname{const}\left(1-\left(\rho_{2}-2 \rho_{1}\right)\right)^{N} .
$$

Thus if the user is confident that the new contour will avoid all singularities by a margin of more than twice the corresponding margin for the previous contour, an immediate change is indicated even if the $(m+1)$ th step was the final step to be made.

If the $m$ th step is an early step in the iteration and $r$ further steps are likely to be carried out, the appropriate comparison is between $E_{1}(N)$ and $E_{2}(N)$. The wasted $2^{m}$ function evaluations are only a small proportion of the total number used. Thus at an early stage, the user should be ready to abandon the contour at the slightest hint that he can make any improvement in the asymptotic behavior of $E$.

For square contours a similar analysis leads to

$$
\frac{E_{1}(2 N)}{E_{2}(N)} \sim \frac{\left(\log _{2} N\right)^{2}}{\pi^{4}\left(\rho_{2} / 2 \rho_{1}\right)^{6}} N^{4 \log _{2}\left(\rho_{2} / 2 \rho_{2}\right)} .
$$

This form is too complicated for any very simple criterion. However, if $\rho_{2}>2 \rho_{1}$, the user might well be advised to change the contour even if this step is the final one.

Of course, if the user knew in advance where the singularities lay, he could choose the optimum contour before starting the integration. In practice, he is more likely to become aware of a nearby singularity as the integration proceeds; but he will not know about other, more distant singularities that may lie near his projected alternative contour. Hence the choice of a new contour will usually be based on incomplete evidence. However, the estimates given here show that it may be worthwhile in case of trouble to try several different contours, and the integration procedures of Paper A have been programmed to take advantage of this possibility.

Oak Ridge National Laboratory

Oak Ridge, Tennessee

1. F. L. Bauer, H. Rutishauser \& E. Stiefel, New aspects in numerical quadrature, Proc. Sympos. Appl. Math., Vol. 15, pp. 199-218, Amer. Math Soc., Providence, R. I., 1963. MR $30 \# 4384$.

2. L. M. Delves \& J. N. Lyness, "A numerical method for locating the zeros of an analytic function," Math. Comp., v. 21, 1967, pp. 543-560.

3. J. N. Lynkss \& C. B. Moler, "Numerical differentiation of analytic functions," SIAM J. Numer. Anal., v. 4, 1967, pp. 202-210.

4. J. N. Lyness, "Differentiation formulas for analytic functions," Math. Comp. (To appear). 